\title{
Bulk Viscosity of Magnetized Neutron Star Matter
}

\author{
J D Anand, V K Gupta, Ashok Goyal, S Singh, Kanupriya Goswami \\ Department of Physics and Astrophysics, \\ University of Delhi,Delhi-11000\%,India. \\ Inter University Centre for Astronomy and Astrophysics \\ Ganeshkhind,Pune-41100\%,India.
}

\begin{abstract}
We study the effect of magnetic field on the bulk viscosity of nuclear matter in neutron stars. We employ the framework of relativistic mean field theory to observe the dense nuclear matter in neutron stars. The effects are first studied for the case when the magnetic field does not exceed the critical value to confine the electrons to the lowest Landau levels. We then consider the case of intense magnetic field to evaluate viscosity for the URCA processes and show that the inequality $p_{F}(e)+p_{F}(p) \geq p_{F}(n)$ is no longer required to be satisfied for the URCA processes to proceed.
\end{abstract}




\section{Introduction}

After the discovery of magnetars there has been a lot of interest in the study of the effects of strong magnetic fields on the various astrophysical phenomena. The observed soft gamma repeaters discovered recently by Kuoveliotou C et al [1] and Kulkarni and Thomson [2] show the presence of strong magnetic fields of upto $10^{15}$ Gauss. Though the core of neutron stars is poorly understood, magnetic fields as strong as $B \sim 10^{18}$ Gauss may be assumed in the core by the scalar virial theorem. It has been argued that even higher fields ( $B \sim 10^{20}$ Gauss) could be consistent with the virial theorem [3]. It is therefore important to investigate the global properties of these objects, particularly a detailed investigation of the stability of dense stellar matter in the presence of ultra strong magnetic fields. This requires a study of the bulk viscosity of such objects, since the damping of vibrations and secular instability of rapidly rotating neutron stars depend upon the bulk viscosity. The bulk viscosity of neutron stars arises mainly due to deviation from $\beta$ equilibrium and the ensuing non-equilibrium reactions. These important reactions are so called URCA processes:

$$
\begin{aligned}
& n \longrightarrow p+e^{-}+\overline{\nu_{e}} \\
& p+e^{-} \longrightarrow n+\nu_{e}
\end{aligned}
$$

and the modified URCA processes:

$$
\begin{aligned}
& n+n \rightarrow n+p+e^{-}+\overline{\nu_{e}} \\
& n+p+e^{-} \rightarrow n+n+\nu_{e}
\end{aligned}
$$

At low temperatures for degenerate nuclear matter, the direct URCA process 
can take place only near the fermi energies of participating particles. Simultaneous conservation of energy and momentum puts the constraint

$$
p_{F}(e)+p_{F}(p) \geq p_{F}(n)
$$

which must be satisfied for the URCA processes to take place. In most models of nuclear matter this constraint is satisfied only at high densities,viz, $n_{b} \geq 1.5 n_{0}$, where $n_{0}=0.16 \mathrm{fm}^{-3}$, is the nuclear saturation density. There have been many studies of the bulk viscosity of neutron stars under the usual situation of zero magnetic field [4-8]. In this note we report the calculations of bulk viscosity of neutron stars from the URCA processes in the presence of magnetic field. Such a study is of interest because significant changes in the equation of state of a magnetized star could lead to similar changes in the bulk viscosity as well. We have considered a wide range of magnetic fields in our study, from "low" to "very high" magnetic fields $\left(\geq 10^{19} G\right)$. As has been discussed above, such extremely strong magnetic fields in the interior of stellar objects can not be completely discounted and have infact been already studied. For the description of nuclear matter at high densities which are found in the interior of neutron stars, we use the frame work of relativistic nuclear mean field theory, including the $\rho$ contribution, as extended to include strong interactions among the particle species involved. As is well known, this would give a fairly good description of strong interactions among the various particles and can be easily extended to include higher resonances as well. In Section 2 we derive expressions for bulk viscosity both in the high and low magnetic field limits. In Section 3 we present our results followed by a brief discussions.

\section{Derivation of Bulk Viscosity}

In this Section we derive expressions for the bulk viscosity in the presence of magnetic field. The presence of magnetic field affects the bulk viscosity only through the changes in the reaction rates as well as through the modification 
of the chemical composition of neutron star matter. We therefore adopt an approach similar to that of Wang and Lu [4], Sawyer [5], Madsen[6] and Gupta et al [7] for the zero magnetic field case. The bulk viscosity $\zeta$ is defined by

$$
\zeta=2\left(\frac{d W}{d t}\right)_{a v} \frac{1}{v_{0}}\left(\frac{v_{0}}{\Delta v}\right)^{2} \frac{1}{\omega^{2}}
$$

Here $v_{0}$ is the specific volume of the star in equilibrium configuration, $\Delta v$ is the amplitude of the periodic perturbation with period $\tau=\frac{2 \pi}{\omega}$ :

$$
v(t)=v_{0}+\Delta v \sin \left(\frac{2 \pi t}{\tau}\right)
$$

The quantity $\left(\frac{d W}{d t}\right)_{a v}$ is the mean dissipation rate of energy per unit mass and is given by the equation

$$
\left(\frac{d W}{d t}\right)_{a v}=-\frac{1}{\tau} \int P(t) \frac{d v}{d t} d t
$$

In the above the pressure $P(t)$ can be expressed near its equilibrium value $P_{0}$, as

$$
P(t)=P_{0}+\left(\frac{\partial P}{\partial v}\right)_{0} \delta v+\left(\frac{\partial P}{\partial n_{p}}\right)_{0} \delta n_{p}+\left(\frac{\partial P}{\partial n_{e}}\right)_{0} \delta n_{e}+\left(\frac{\partial P}{\partial n_{n}}\right)_{0} \delta n_{n}
$$

The change in the number of neutrons, protons and electrons per unit mass over a time interval $(0, t)$ due to the URCA reactions (1) and (2) is given by

$$
-\delta n_{n}=\delta n_{p}=\delta n_{e}=\int_{0}^{t} \frac{d n_{p}}{d t} d t
$$

The net rate of production of protons, $\frac{d n_{p}}{d t}$ is given by the difference between the rates $\Gamma_{1}$ and $\Gamma_{2}$ of reactions (1) and (2) respectively. At equilibrium the two rates are obviously equal and the chemical potentials satisfy the equality

$$
\Delta \mu=\mu_{n}-\mu_{p}-\mu_{e}=0
$$

A small volume perturbation brings about a small change in the chemical potentials and the above inequality is no longer satisfied, now $\Delta \mu$ is not zero and consequently the reaction rates of the processes (1) and (2) are no longer equal. The net rate of production of protons will thus depend upon the value 
of $\Delta \mu$. In the linear approximation, $\frac{\Delta \mu}{k T} \ll 1$, the net rate is proportional to $\Delta \mu$ and thus can be written as

$$
\frac{d n_{p}}{d t}=\Gamma_{1}-\Gamma_{2}=-\lambda \Delta \mu
$$

Using the thermodynamic relation

$$
\frac{\partial P}{\partial n_{i}}=-\frac{\partial \mu_{i}}{\partial v}
$$

in equation (9) and employing equation (10), we obtain

$$
\begin{aligned}
\delta P= & P(t)-P_{0} \\
& =-\frac{\partial(\delta \mu)}{\partial v} \int_{0}^{t} \lambda \delta \mu(t) d t
\end{aligned}
$$

The change in the chemical potential $\delta \mu(t)$ arises due to a change in the specific volume $\delta v$ and changes in the concentrations of various species, viz, neutrons, protons and electrons. Thus

$$
\delta \mu(t)=\delta \mu(0)+\left(\frac{\partial \delta \mu}{\partial v}\right)_{0} \delta v+\left(\frac{\partial \delta \mu}{\partial n_{n}}\right)_{0} \delta n_{n}+\left(\frac{\partial \delta \mu}{\partial n_{p}}\right)_{0} \delta n_{p}+\left(\frac{\partial \delta \mu}{\partial n_{e}}\right)_{0} \delta n_{e}
$$

Using equations (10)-(12), we arrive at the following equations for $\delta \mu$ :

$$
\frac{d \delta \mu}{d t}=\omega A \frac{\Delta v}{v_{0}} \cos (\omega t)-C \lambda \delta \mu
$$

where

$$
\begin{gathered}
A=v_{0}\left(\frac{\partial \delta \mu}{\partial v}\right)_{0} \\
C=v_{0}\left(\frac{\partial \delta \mu}{\partial n_{p}}+\frac{\partial \delta \mu}{\partial n_{e}}-\frac{\partial \delta \mu}{\partial n_{n}}\right)_{0}
\end{gathered}
$$

Since for small perturbations, $\lambda, \mathrm{A}$ and $\mathrm{C}$ are constants, equatioin (15) can be solved analytically to give

$$
\delta \mu=\frac{\omega A}{\omega^{2}+C^{2} \lambda^{2}} \frac{\Delta v}{v_{0}}\left[-C \lambda e^{-C \lambda t}+\omega \sin (\omega t)+C \lambda \cos (\omega t)\right]
$$

Finally using equations (6), (8), (10), (11), (14) and (17)-(19), we obtain the following expressions for $\zeta$

$$
\zeta=\frac{A^{2} \lambda}{\omega^{2}+C^{2} \lambda^{2}}\left[1-\frac{\omega C \lambda}{\pi} \frac{1-e^{-C \lambda \tau}}{\omega^{2}+C^{2} \lambda^{2}}\right]
$$


Given the number densities of the these particle species in terms of their respective chemical potentials, one can determine the coefficients $\mathrm{A}$ and $\mathrm{C}$; given the rates $\Gamma_{1}$ and $\Gamma_{2}$ for the two processes (1) and (2) one can determine $\lambda$ and hence $\zeta$ for any given baryon density and temperature. For the description of the dense nuclear matter we have used Walecka's mean field theory [8-10]. In the mean field approximation we allow the mesons fields $\sigma, \omega$ and $\rho$ to acquire density dependent average values. From the effective langrangian one can then read off the effective masses and chemical potentials $\bar{m}_{i}$ and $\bar{\mu}_{i}$. The fermi momenta, are in turn related to these effective quantities by

$$
\bar{k}_{i}=\sqrt{\bar{\mu}_{i}^{2}-\bar{m}_{i}^{2}}
$$

The various chemical potentials and masses required in these expressions are obtained from the self consistent solution of the mean field equations:

$$
\begin{array}{r}
m_{\sigma}^{2} \bar{\sigma}+b m_{n} b_{\sigma N}^{3} \bar{\sigma}^{2}+c g_{\sigma N}^{4} \bar{\sigma}^{3}-g_{\sigma N}\left(n_{p}^{s}+n_{n}^{s}\right)=0 \\
m_{\omega}^{2} \bar{\omega}_{0}=g_{\omega N}\left(n_{p}+n_{n}\right) \\
m_{\rho}^{2} \bar{\rho}_{0}=\frac{1}{2} g_{\rho N}\left(n_{p}-n_{n}\right) \\
m_{n}-\bar{m}_{n}=m_{p}-\bar{m}_{p}=g_{\sigma n} \bar{\sigma}_{0} \\
\bar{\mu}_{n}=\mu_{n}-g_{\omega N} \bar{\omega}_{0}+\frac{1}{2} g_{\rho N} \bar{\rho}_{0} \\
\bar{\mu}_{p}=\mu_{p}-g_{\omega N} \bar{\omega}_{0}-\frac{1}{2} g \rho N \bar{\rho}_{0}
\end{array}
$$

The number densities $n_{i}$ and the scalar densities $n_{i}^{s}$ [9] are given in terms of these quantities by the expressions

$$
\begin{gathered}
n_{i}=2 \int \frac{d^{3} p}{(2 \pi)^{3}}\left(1+e^{\frac{E_{i}-\bar{\mu}_{i}}{T}}\right)^{-1} \\
n_{i}^{s}=2 \bar{m}_{i} \int \frac{d^{3} p}{(2 \pi)^{3}} \frac{1}{E_{i}}\left(1+e^{\frac{E_{i}-\bar{\mu}_{i}}{T}}\right)^{-1}
\end{gathered}
$$

where

$$
E_{i}=\sqrt{p^{2}+\bar{m}_{i}^{2}}
$$


Since the chemical potentials $\mu_{n}$ and $\mu_{p}$ are of the order of a few hundred $\mathrm{MeV}$, even for temperatures upto a few $\mathrm{MeV}$ the matter is completely degenerate, this leads to the following expressions for $n_{i}$ and $n_{i}^{s}$ :

$$
\begin{gathered}
n_{i}=\frac{1}{3 \pi^{2}}\left(\bar{\mu}_{i}-\bar{m}_{i}^{2}\right)^{\frac{3}{2}} \\
n_{i}^{s}=\frac{\bar{m}_{i}}{2 \pi^{2}} \bar{\mu}_{i}\left(\bar{\mu}_{i}^{2}-\bar{m}_{i}^{2}\right)^{\frac{1}{2}}
\end{gathered}
$$

where $\mathrm{i}=\mathrm{n}, \mathrm{p}$. To evaluate $n_{e}$, set $\bar{\mu}_{e}=\mu_{e}$ and $\bar{m}_{e}=m_{e}$ in equation(26).

It now remains to introduce the magnetic field. In the presence of constant magnetic field B in the z-direction, the energy of a charged particle of mass $\mathrm{m}$ and charge e is given by

$$
E^{2}=p_{z}^{2}+m^{2}+2 \nu e B
$$

where the quantum number $\nu$ is given by

$$
\nu=\nu_{L}+\frac{1}{2}+\sigma
$$

for the Landau level $\nu_{L}=0,1,2 \ldots$ and $\operatorname{spin} \sigma= \pm \frac{1}{2}$.

In the presence of a magnetic field, the number densities of electrons and protons are given by

$$
n_{e}=\frac{e B v}{2 \pi^{2}} \sum_{\nu=0}^{\nu_{\max }}\left(2-\delta_{\nu, 0}\right) \sqrt{\mu_{e}^{2}-m_{e}^{2}-2 \nu e B}
$$

and

$$
n_{p}=\frac{e B v}{2 \pi^{2}} \sum_{\nu=0}^{\nu_{\max }}\left(2-\delta_{\nu, 0}\right) \sqrt{\bar{\mu}_{p}^{2}-\bar{m}_{p}^{2}-2 \nu e B}
$$

whereas the neutron number density expression remains unchanged namely

$$
n_{n}=\frac{1}{3 \pi^{2}}\left(\bar{\mu}_{n}^{2}-\bar{m}_{n}^{2}\right)^{\frac{3}{2}}
$$

In the decay rates the usual sum over the charged particle states (per unit volume) in zero field, viz $\frac{2}{h^{3}} \int d^{3} p$ is replaced by $\sum_{\nu}\left(2-\delta_{\nu, 0}\right) \int_{-\infty}^{\infty} d p_{z} \int_{-\frac{e B L_{x}}{2}}^{\frac{e B L_{x}}{2}} d p_{y}$ and the matrix elements have to be calculated by using the exact wave functions for electrons and protons obtained by solving the Dirac equation in the presence of magnetic field [11-15]. We are now in a position to evaluate the various quatities required for the calculation of bulk viscosity, viz, A, C and $\lambda$. 


\subsection{Weak Magnetic Field}

For weak magnetic field several Landau levels are populated and the matrix elements remain essentially unchanged [13-14] and one needs to account for the correct phase space factor. For non-relativistic degenerate nucleons the decay rate constant $\lambda$ is given by

$$
\begin{aligned}
\lambda= & \frac{17}{480 \pi} G_{F}^{2} \cos _{\theta_{c}}^{2} T^{4}\left(1+3 g_{A}^{2}\right) e B \bar{m}_{p} \bar{m}_{n} \theta\left(p_{F}(p)+p_{F}(e)-p_{F}(n)\right) \\
& \sum_{\nu=0}^{\nu_{\max }}\left[2-\delta_{\nu, 0}\right] \frac{1}{\sqrt{\mu_{e}^{2}-m_{e}^{2}-2 \nu e B}}
\end{aligned}
$$

which reduces to well known expressions [16-17] in the limit $B \rightarrow 0$. Using equations (30),(31) and (32) the coefficients $\mathrm{A}$ and $\mathrm{C}$ given in equations (17) and (18) become

$$
\begin{aligned}
A= & {\left[-\frac{\left(\bar{\mu}_{n}^{2}-\bar{m}_{n}^{2}\right)}{3 \bar{\mu}_{n}}+\frac{\sum_{\nu=0}^{\nu_{\max }}\left(2-\delta_{\nu, 0}\right) \sqrt{\bar{\mu}_{p}^{2}-\bar{m}_{p}^{2}-2 \nu e B}}{\sum_{\nu=0}^{\nu_{\max }}\left(2-\delta_{\nu, 0}\right) \bar{\mu}_{p}\left(\bar{\mu}_{p}^{2}-\bar{m}_{p}^{2}-2 \nu e B\right)^{-\frac{1}{2}}}\right.} \\
& \left.\frac{\sum_{\nu=0}^{\nu_{\max }}\left(2-\delta_{\nu, 0}\right) \sqrt{\mu_{e}^{2}-m_{e}^{2}-2 \nu e B}}{\sum_{\nu=0}^{\nu_{\max }}\left(2-\delta_{\nu, 0}\right) \mu_{e}\left(\mu_{e}^{2}-m_{e}^{2}-2 \nu e B\right)^{-\frac{1}{2}}}\right]-\frac{1}{2}\left(\frac{g_{\rho N}}{m_{\rho}}\right)^{2}\left(n_{p}-n_{n}\right) \\
C= & {\left[\frac{\pi^{2}}{\bar{\mu}_{n}^{2} \sqrt{\bar{\mu}_{n}^{2}-\bar{m}_{n}^{2}}}+\frac{2 \pi^{2}}{e B} \frac{1}{\sum_{\nu=0}^{\nu_{\max }}\left(2-\delta_{\nu, 0}\right) \bar{\mu}_{p}\left(\bar{\mu}_{p}^{2}-\bar{m}_{p}^{2}-2 \nu e B\right)^{-\frac{1}{2}}}\right.} \\
& \left.\frac{2 \pi^{2}}{e B} \frac{1}{\sum_{\nu=0}^{\nu_{\max }}\left(2-\delta_{\nu, 0}\right) \mu_{e}\left(\mu_{e}^{2}-m_{e}^{2}-2 \nu e B\right)^{-\frac{1}{2}}}\right]+\left(\frac{g_{\rho N}}{m_{\rho}}\right)^{2}
\end{aligned}
$$

\section{$2.2 \quad$ Strong Magnetic Field}

For strong magnetic field, the electron is forced into the lowest Landau level corresponding to $\nu=0$. This happens for a field $B>B_{c}$, where $B_{c}$ is given by $e B_{c}=\frac{\mu_{e}^{2}-m_{e}^{2}}{2}$. Because of the equality $n_{p}=n_{e}$, dictated by charge neutrality, it is then straight forwad to verify that for $B>B_{c}$ only the lowest Landau level $\nu=$ 0 contributes for the protons as well. This leads to considerable simplification in the expression for the decay rate. Using the exact wave functions for protons 
and electrons in the lowest Landau level and following [15], the rate for the process (1) can be calculated and we get

$$
\begin{aligned}
\Gamma= & \frac{2 G_{F}^{2} \cos _{\theta_{c}}^{2}}{(2 \pi)^{5}} e B \frac{\bar{m}_{n} \bar{m}_{p}}{p_{F}(p)} \int d E_{p} d E_{e} d E_{n} d E_{\nu} E_{\nu}^{2} \delta\left(E_{p}+E_{e}-E_{n}-E_{\nu}\right)\left(1-f_{n}\right) f_{p} f_{e} \\
& {\left[4 g_{A}^{2}+\left(g_{A}+g_{v}\right)^{2}\right]\left[e^{-\frac{\left(p_{F}^{2}(n)-4 p_{F}^{2}(e)\right)}{2 e B}} \theta\left(p_{F}^{2}(n)-4 p_{F}^{2}(e)\right)+e^{-\frac{p_{F}^{2}(n)}{2 e B}} \theta\left(p_{F}(n)\right)\right](36) }
\end{aligned}
$$

Carrying out the energy integrals for degenerate matter, the decay constant $\lambda$ is given by

$$
\begin{aligned}
\lambda= & \frac{17}{960 \pi} G_{F}^{2} \cos _{\theta_{c}}^{2} e B \frac{\bar{m}_{n} \bar{m}_{p}}{p_{F}(p)} T^{4}\left[4 g_{A}^{2}+\left(g_{v}+g_{A}\right)^{2}\right] \\
& {\left[e^{-\frac{\left(p_{F}^{2}(n)-4 p_{F}^{2}(e)\right)}{2 e B}} \theta\left(p_{F}^{2}(n)-4 p_{F}^{2}(e)\right)+e^{-\frac{p_{F}^{2}(n)}{2 e B}} \theta\left(p_{F}(n)\right)\right] }
\end{aligned}
$$

The constants $\mathrm{A}$ and $\mathrm{C}$ are obtained from equations (34) and (35) by setting $\nu=0$. Substituting A, C and $\lambda$ into the expression (20) the bulk viscosity can now be calculated for a given baryon density and temperature.

\section{$3 \quad$ Results and Discussions}

In this paper we have used the relativistic mean field approximation to describe nuclear matter at high densities. This approach gives a good description of nuclear matter and neutron star properties at high densities. We have used the values of the various couplings $g_{\sigma N}, g_{\omega N}, g_{\rho N}$, b and c given in [10] namely

$$
\begin{array}{r}
\frac{g_{\sigma N}}{m_{\sigma}}=0.0152502 \mathrm{MeV}^{-1} \\
\frac{g_{\omega N}}{m_{\omega}}=0.011 \mathrm{MeV}^{-1} \\
\frac{g_{\rho N}}{m_{\rho}}=0.011 \mathrm{MeV}^{-1} \\
b=3.418 \times 10^{-3} \\
c=0.0146
\end{array}
$$

With this set of parameters, one obtains $n_{B}=0.153 \mathrm{fm}^{-3}$; binding energy $\left(\frac{E}{n_{B}}-m_{n}\right)=-16.3 \mathrm{MeV}$; charge symmetry energy $=32.5 \mathrm{MeV}$; bulk modulous $=300 \mathrm{MeV}$ and Landau mass $=0.83 m_{N}$. 
We have computed the bulk viscosity for zero magnetic field, for low magnetic fields by summing over various Landau levels and finally for very high magnetic fields, where only the $\nu=0$ level contributes. In figure 1 we show the variation of the coefficient of bulk viscosity with baryon density for $e B=0$ and $e B=10^{4} \mathrm{MeV}^{2}$ for $T=1 \mathrm{MeV}$. As can be seen from (33) and (36) the variation of $\zeta$ with $\mathrm{T}$ goes as $T^{4}$; so for other temperatures $\zeta$ can be obtained by scaling. We, in our study, have divided the magnetic field into two regimes, the "low" magnetic field where a large number of Landau levels contribute and the "strong" magnetic field where only the lowest Landau level contributes. The critical field $B_{c}$ separates the two regimes depends crucially on the chemical potential of the electron/proton. These in turn depend upon the number density $n_{B}$. As $n_{B}$ varies from $0.2 \mathrm{fm}^{-3}$ to $2.0 \mathrm{fm}^{-3}$, the range of $n_{B}$ over which figure 1 is plotted, $e B_{c}$ remains well above $10^{4} \mathrm{MeV}^{2}$. Therefore we have used the weak field formula for computing viscosity at this value of magnetic field. At zero magnetic field the viscosity changes smoothly with $n_{B}$. As $n_{B}$ changes from $0.2 \mathrm{fm}^{-3}$ to $2.0 \mathrm{fm}^{-3}$ viscosity changes by nearly one order of magnitude. At $e B=10^{4} \mathrm{MeV}^{2}$, superimposed on a nearly smooth change is a rapid fluctuation in viscosity with $n_{B}$. As one can see, whenever a new Landau level appears, the reaction rate shows a sudden discontinous change which is reflected in a rapid fluctuation in viscosity. In figure 2 , we show the variation of viscosity with $n_{B}$ for "high" magnetic fields. This is achieved by considering only very small number densities, viz, $n_{B} \leq 0.01 \mathrm{fm}^{-3}$. For such small $n_{B}$, even at magnetic fields of a few hundreds $M e V^{2}$, only the lowest Landau level contributes and therefore one can use the formulas obtained in the high magnetic field case. The direct URCA process is now no longer inhibited whereas for $B=0$ the process is not allowed by energy momentum conservation and the bulk viscosity does not get any contribution. We find that the viscosity decreases very rapidly with the changes in number density. For example, for $e B=100 \mathrm{MeV}^{2}$, the viscosity changes by 12 orders of magnitude as $n_{B}$ varies from $0.001 \mathrm{fm}^{-3}$ to $0.004 \mathrm{fm}^{-3}$. The variation in viscosity with $n_{B}$ is most drastic at lower magnetic fields; at higer magnetic fields the variation with $n_{B}$ 
becomes much less, so much so that for $e B=1000 \mathrm{MeV}^{2}$ the bulk viscosity remains almost constant and beyond that, it infact starts increasing with $n_{B}$. In figure 3 we plot bulk viscosity against the magnetic field for two baryon densities,viz, $n_{B}=0.34 \mathrm{fm}^{-3}$ and $n_{B}=0.6 \mathrm{fm}^{-3}$. For magnetic fields upto $10,000 \mathrm{MeV}^{2}$, the weak field approximation is valid as pointed out earlier. Here we find, not surprisingly, that the viscosity is nearly independent of magnetic field. As the magnetic field increases (beyond $10^{4} \mathrm{MeV}^{2}$ for $n_{B}=0.34 \mathrm{fm}^{-3}$ and $3 \times 10^{4} \mathrm{MeV}^{2}$ for $n_{B}=0.6 \mathrm{fm}^{-3}$ ) bulk viscosity begins to increase with magnetic field because now only the lowest Landau level contributes and the magnetic field can be considered "high". In figure 4, we show the variation of viscosity with magnetic field at low densities. We find that initially the viscosity increases very rapidly but then saturates to a nearly constant value as the magnetic field increases. To conclude we find that in the presence of strong magnetic field, the triangular inequality is no longer required to be satisfied for the URCA process to contribute to the viscosity. At very low densities this requirement can be met for magnetic field strengths of a few hundred $\mathrm{MeV}^{2}$, and the bulk viscosity of the medium is finite though small. This is to be contrasted with the situation in which the magnetic field is zero and the URCA process does not contribute to bulk viscosity upto a certain minimum density. However at densities of relevance in neutron $\operatorname{stars}\left(n_{B} \sim 2-4 n_{0}\right)$, the effect of non-zero magnetic field will be felt only at extremely high values of $\mathrm{B}\left(\geq 10^{4} \sim 10^{5}\right)$. even though the reaction rate is directly proportional to the magnetic field, the viscosity depends in a rather complicated fashion on the magnetic field. 


\section{References}

[1] Kuoveliotou et al, Nature 393, 215(1998)

[2] Kulkarni S R and Thomson C, Nature 393, 215 (1998)

[3] Deog Ki Hong, Phys. Lett. B 445, 36(1998)

[4] Q D Wang and T Lu, Phys. Rev. Lett B148, 211,(1984)

[5] R F Sawyer, Phys. Rev. D 39, 3804(1989)

[6] Jes Madsen, Phys. Rev. D 46, 3290(1992)

[7] V K Gupta, A Wadhwa, S Singh and J D Anand, Pramana-J Phys.49, 443(1997)

[8] J D Walecka, Ann. Phys 83, 491(1974)

[9] B D Serot and J D Walecka, Advances in nuclear Physics 16, 1(1986)

[10] J Ellis, J I Kapusta and K A Olive, Nucl. Phys 348, 345(1991)

[11] D Lai and S L Shapiro, Astrophys. J 383, 745(1991)

[12] S Chakarvarty, D Bandhopadhyay and S Pal, Phys. Rev. Lett 78, 2898(1997)

[13] J J Matese and R F O'Connell, Astrophys. J160, 739(1970)

[14] B Cheng, D N Schramn and J W Truran Phys. Lett. B 316, 521(1993)

[15] Ashok Goyal, V K Gupta, Kanupriya Goswami and Vinita Tuli, hep-ph 9812494

[16] J M Lattimer, C J Pethik, M Prakash and P Haensel, Phys. Rev. Lett 66, 2701(1991)

[17] P Haensel and R Schaeffer, Phys. Rev. D 45, 4708(1992) 


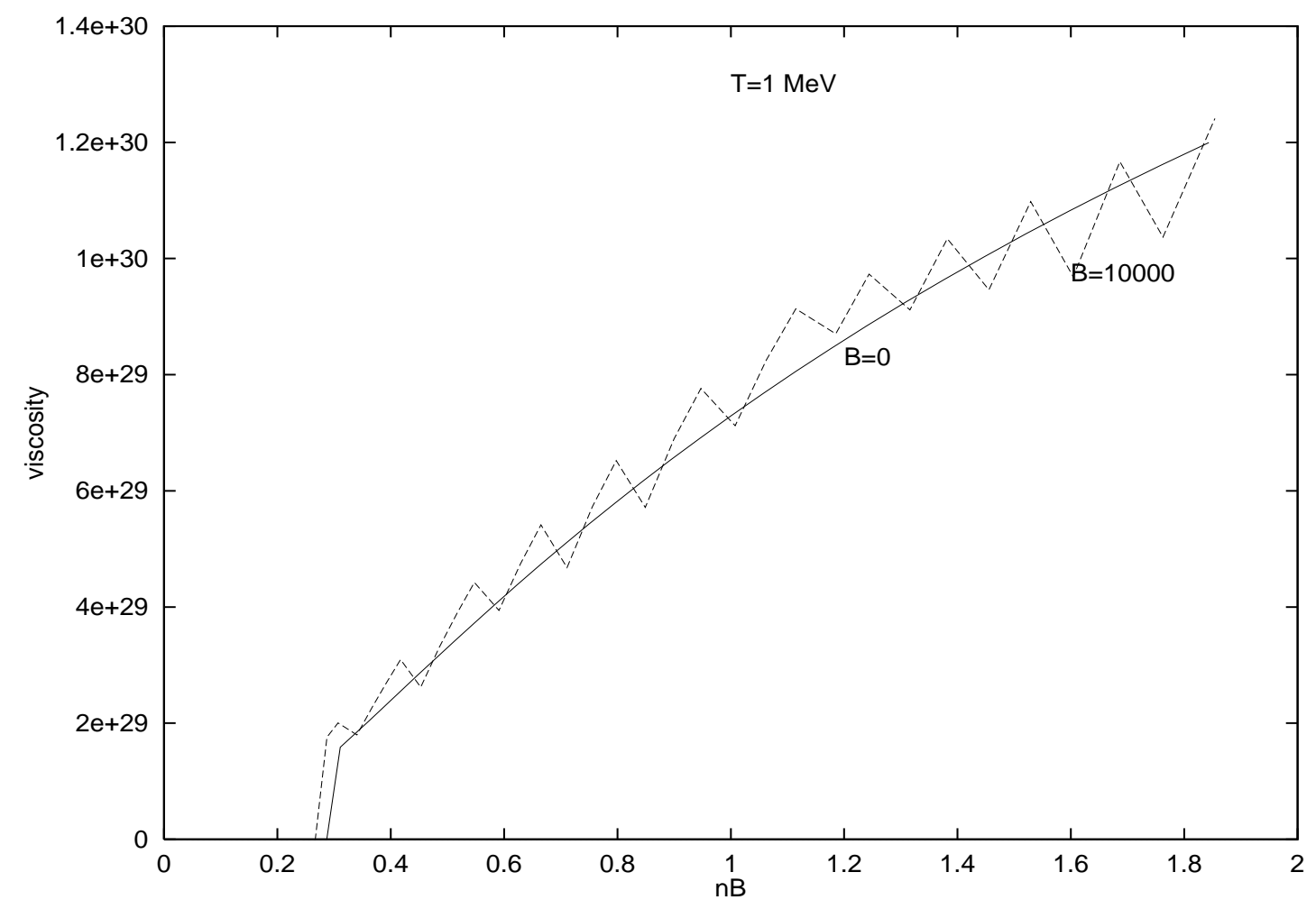

Figure 1

Figure 1: Bulk Viscosity $\zeta$ in units of $\mathrm{gm} . \mathrm{cm} . \mathrm{s}^{-1}$ as a function of Baryon Density $n_{B}$ in $\mathrm{fm}^{-3}$ at $T=1 \mathrm{MeV}$ for magnetic field $e B=0$ and $e B=10^{4} \mathrm{MeV}^{2}$. 


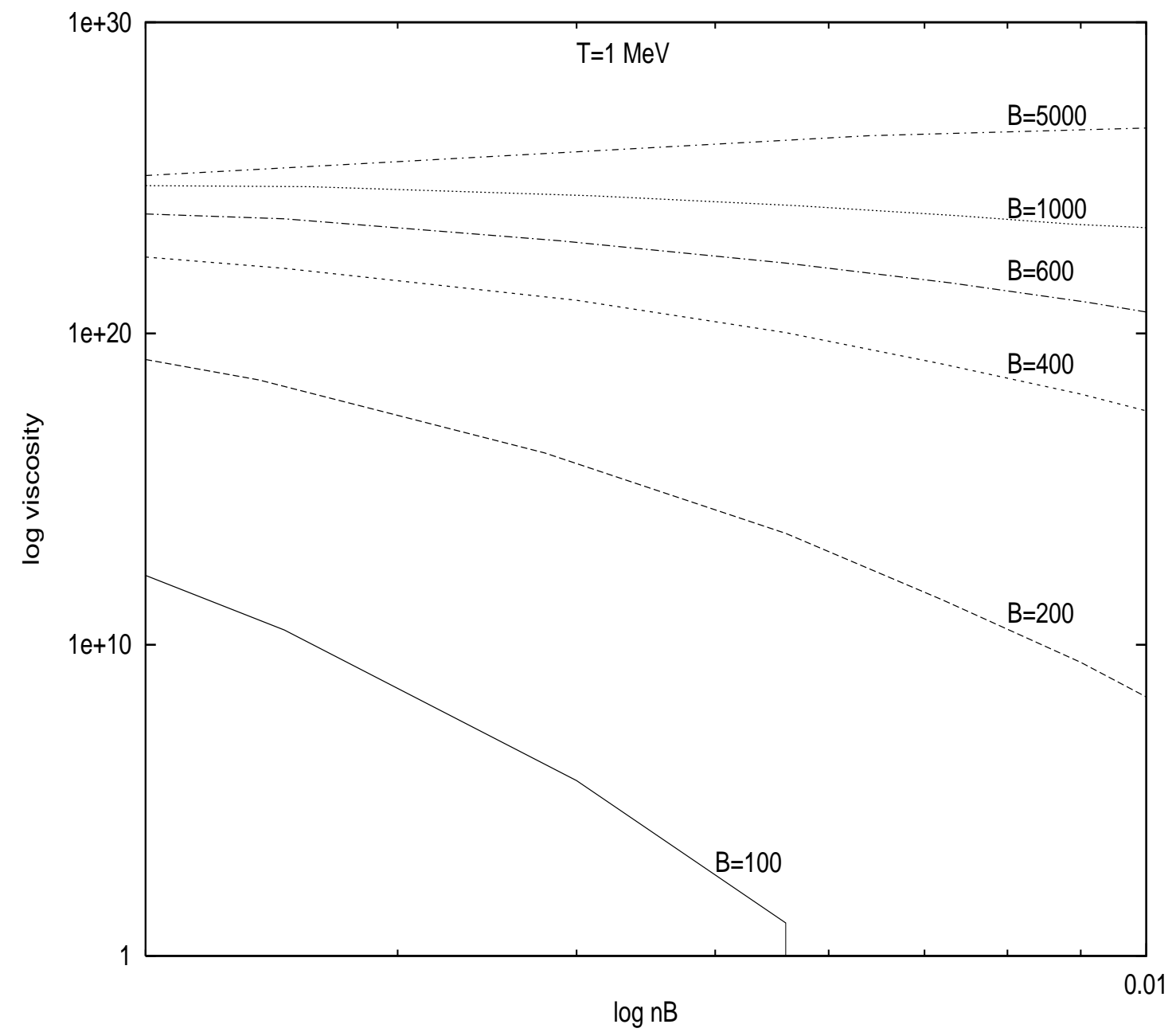

Figure 2

Figure 2: Bulk Viscosity $\zeta$ as a function of Baryon Density $n_{B}$ for different values of the magnetic field at $T=1 \mathrm{MeV}$. The units of $\zeta, n_{B}$ and $\mathrm{B}$ are same as in Fig. 1. 


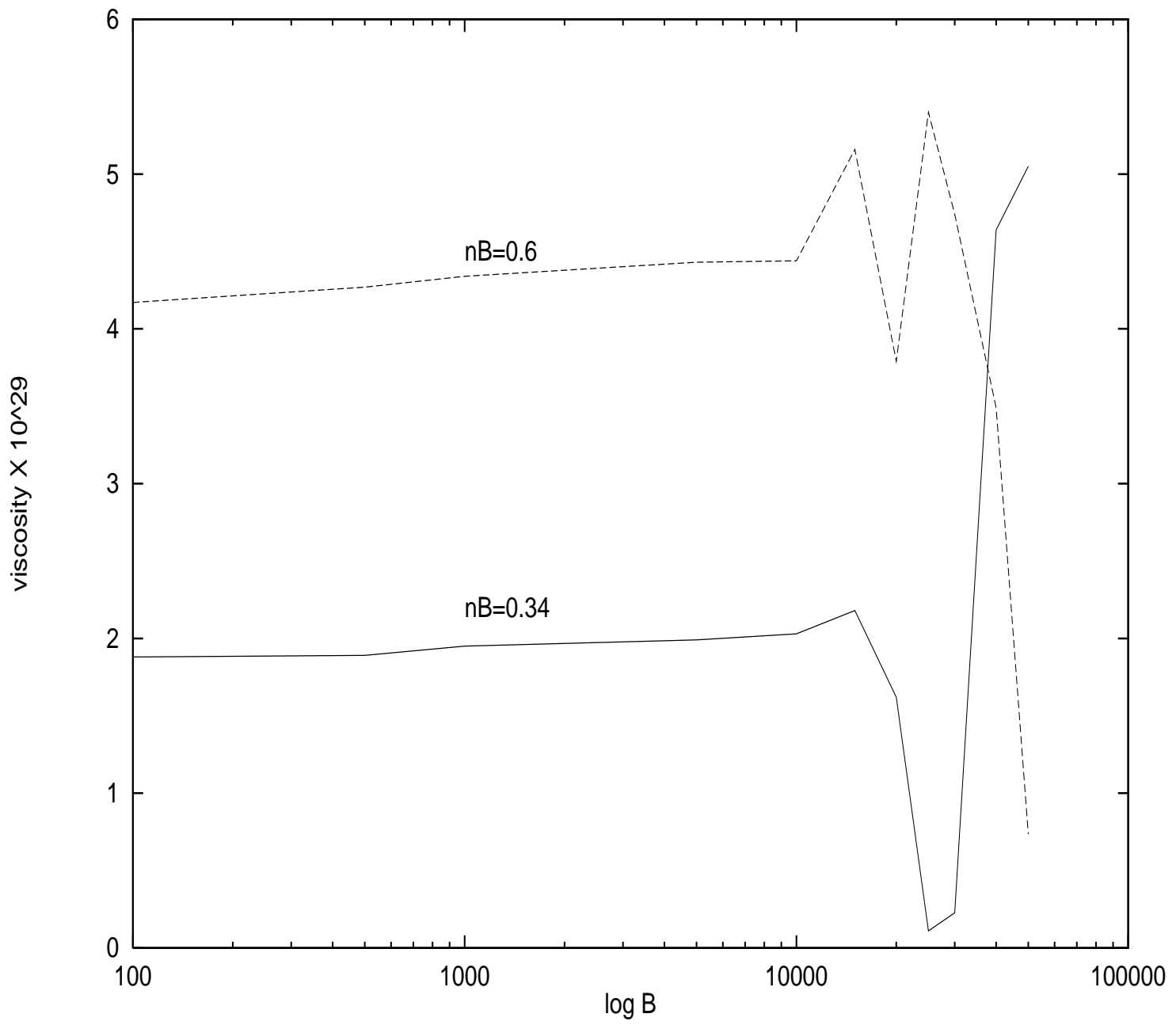

Figure 3

Figure 3: Bulk Viscosity $\zeta$ as a function of magnetic field at $T=1 \mathrm{MeV}$ for different values of baryon density. The units of $\zeta, n_{B}$ and $\mathrm{B}$ are same as in Fig. 1. 


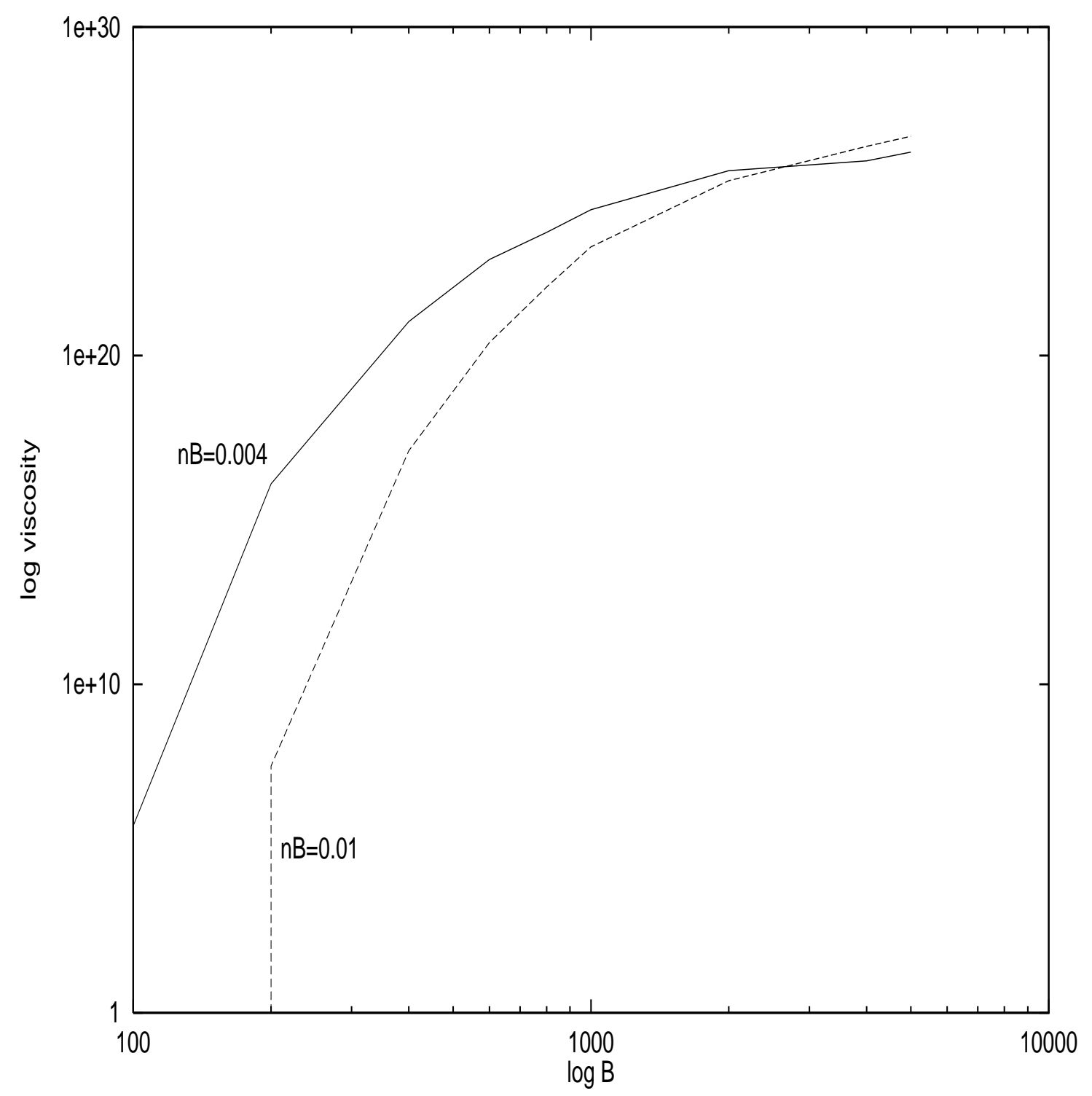

Figure 4

Figure 4: Bulk Viscosity $\zeta$ as a function of magnetic field at $T=1 \mathrm{MeV}$ for different values of baryon density. The units of $\zeta, n_{B}$ and $\mathrm{B}$ are same as in Fig. 1. 